\title{
Perfis de Vinculação, Coparentalidade e Ajustamento Familiar em Pais Recém-Divorciados: Diferenças no Ajustamento Psicológico
}

\author{
Attachment, Coparenting and Family Adjustment Profiles in Newly \\ Divorced Parents: Differences in Psychological Adjustment
}

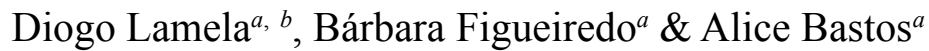 \\ ${ }^{a}$ Universidade do Minho, Braga, Portugal \\ $\&{ }^{b}$ Instituto Politécnico de Viana do Castelo, Viana de Castelo, Portugal
}

\begin{abstract}
Resumo
O objectivo deste estudo foi identificar, através de uma análise de cluster, perfis de regulação individualdiádica-sistémica em pais récem-divorciados $(N=81)$ com base na vinculação, coparentalidade e ajustamento familiar. Três padrões de regulação foram identificados: regulado-seguro (RS), desregulado-ansioso (DA) e desregulado-evitante (DE). O grupo RS mostrou níveis mais elevados de segurança na vinculação, maior qualidade na coparentalidade e no ajustamento familiar que os grupos DA e DE. A coparentalidade e o funcionamento familiar foram as dimensões que melhor diferenciaram os clusters. Avaliando o ajustamento psicológico dos pais recém-divorciados em função dos perfis encontrados, o grupo RS apresentou maiores níveis de ajustamento psicológico do que os restantes dois grupos. Não foram encontradas diferenças no ajustamento psicológico entre os grupos DA e DE.

Palavras-chave: Divórcio, vinculação, coparentalidade, ajustamento familiar, ajustamento psicológico.
\end{abstract}

\begin{abstract}
The aim of this study was to identify, using a cluster analysis, profiles of individual-dyadic-systemic regulation in newly divorced parents $(N=81)$. Three profiles of regulation were identified: secure/regulated (SR), anxious/dysregulated (AD) and avoidant/dysregulated (ED). The SR group reported higher levels of attachment security and high levels of perceived parenting alliance and family adjustment than $\mathrm{AD}$ and ED groups. Coparenting and family functioning were the dimensions that better discriminate between clusters. Assessing the newly divorced parents' psychological adjustment using the three profiles found, the parents of SR group showed higher levels of psychological adjustment than the ones in the other two groups. There were no differences in psychological adjustment between the AD and ED groups.

Keywords: Divorce, attachment, coparenting, family adjustment, psychological adjustment.
\end{abstract}

A investigação tem demonstrado que o ajustamento psicológico dos pais é um dos principais preditores do funcionamento familiar após a dissolução conjugal (Lansford, 2009). Considerando estes dados, o pressuposto teórico testado centra-se na premissa que os pais, enquanto elementos estruturantes das relações familiares, influenciam o funcionamento e desenvolvimento de todos os membros da família, em especial dos filhos. Esta associação entre o ajustamento psicológico parental e o funcionamento familiar parece ser mediada. A literatura sugere que a qualidade da coparentalidade, o nível de conflito interparental, a segurança da vinculação e a qualidade das relações pai/mãe-criança podem funcionar como os principais mediadores familiares do funciona-

*Endereço para correspondência: Escola de Psicologia, Universidade do Minho, Campus de Gualtar, Braga, Portugal, 4710-057. E-mail: dlamela@ese.ipvc.pt mento das crianças (Nunes-Costa, Lamela, \& Figueiredo, 2009; Raposo et al., 2011).

O ajustamento psicológico dos filhos é na sua maioria explicado pelas estrutura e processo familiar. No entanto, pouca relevância é dada a estas mesmas variáveis para a compreensão do ajustamento psicológico dos pais ao divórcio, estudando-se, quase exclusivamente, variáveis internas dos pais (e.g., estratégias de coping, vinculação ao cônjuge e nível educacional) e variáveis externas à família (e.g., redes de suporte social, novas relações íntimas e qualidade e estabilidade na carreira) na compreensão e predição do ajustamento psicológico (Gähler, 2006). Devido à natureza de interdependência dinâmica dentro do sistema familiar, não é errado inferir-se que, tal como nas crianças, as estruturas e processos familiares podem influenciar o ajustamento psicológico dos pais.

No presente estudo, serão testados perfis de regulação do processo de divórcio, em função da vinculação, copa- 
rentalidade e funcionamento familiar. Diferenças entre os perfis serão examinadas ao nível do ajustamento psicológico e ao nível das características sócio-demográficas dos pais.

\section{Factores Individuais, Diádicos e Sistémicos no Ajustamento Psicológico dos Adultos ao Divórcio}

Lamela, Figueiredo e Bastos (2010) conceptualizaram o divórcio como um processo de reorganização da vinculação adulta. Na idade adulta, a vinculação tem como função a protecção e a segurança perante momentos de distress (Mikulincer \& Shaver, 2007; Shaver \& Mikulincer, 2007). A figura de vinculação funciona como prestador de cuidados instrumentais e emocionais, auxiliando a reorganização psicológica da figura vinculada (Feeney \& Monin, 2008). Sendo estrutural e processualmente mais complexo do que na infância, o sistema de vinculação na idade adulta pode ser activado perante três fontes de distress: ameaça real ou percebida ao self, ameaça real ou percebida à relação de proximidade com a figura de vinculação ou, finalmente, outras situações desafiadoras que impulsionam a pessoa procurar os cuidados da figura de vinculação (Mikulincer \& Shaver, 2007). De facto, o divórcio congrega a activação simultânea destas fontes de distress, o que poderá sugerir que o padrão psicológico durante o período após a dissolução conjugal se caracterize como um momento de activação permanente do sistema de vinculação (Davis, Shaver, \& Vernon, 2003). Consequentemente, o ajustamento psicológico após o divórcio pode estar negativamente associado a saliência e frequência dos comportamentos de vinculação ao ex-cônjuge (Kitson, 1982; Tschann, Johnston, \& Wallerstein, 1989) e positivamente relacionado com um estilo de vinculação seguro e, parcialmente, com um estilo de vinculação inseguro-evitante, sendo que adultos com vinculação insegura-ansioso apresentam maior risco para maiores níveis de desajustamento psicológico (BakermansKranenburg \& van IJzendoorn, 1997; Davis et al., 2003; Yárnoz-Yaben, 2010a).

A coparentalidade, por seu turno, é um processo familiar que tem sido alvo de uma crescente investigação empírica na última década (McHale \& Lindahl, 2011). A coparentalidade é definida como a relação mantida entre dois adultos - normalmente a mãe e o pai-que partilham a responsabilidade na educação e na prestação de cuidados instrumentais e emocionais a pelo menos uma criança (Feinberg, 2003; Lamela, Nunes-Costa, \& Figueiredo, 2010). Em consequência, a coparentalidade tem sido identificada empiricamente como um dos principais preditores do ajustamento psicológico das crianças e pais e do funcionamento familiar, quer em famílias intactas, quer em famílias com pais divorciados ou separados (Dorsey, Forehand, \& Brody, 2007; Madden-Derdich, Leonard, \& Christopher, 1999; Schoppe, Mangelsdorf, \& Frosch, 2001; Teubert \& Pinquart, 2010). Conceptualmente associada ao subsistema executivo do sistema familiar (Minuchin, 1974), a coparentalidade é um subsistema autónomo dos subsistemas conjugal e parental. Enquanto o subsistema parental define a relação que cada pai mantém individualmente com a criança (operacionalizada nos estilos e práticas parentais), o subsistema coparental caracteriza a relação dinâmica interparental no cuidado da criança (e.g., Schoppe-Sullivan, Mangelsdorf, Frosh, \& McHale, 2004; van Egeren, 2004).

Adicionalmente, a investigação teórica e empírica têm filtrado um conjunto de componentes que operacionalizam a coparentalidade, tais como o acordo/solidariedade nos valores, princípios e regras de educação da criança, a divisão do trabalho, o conflito interparental, a proximidade emocional, o suporte/sabotagem da parentalidade e a gestão conjunta da família (Feinberg, 2003; Teubert \& Pinquart, 2010; van Egeren, 2003; para revisão Lamela, Nunes-Costa, et al., 2010). A variação no conjunto destes componentes parece explicar o peso da coparentalidade na predição do funcionamento individual dos pais, das crianças e das famílias (Feinberg, 2003; McHale \& Irace, 2011). Apesar de uma forma não consistente, estudos anteriores têm demonstrado que a qualidade coparentalidade está positivamente associada a características sócio-demográficas dos pais, nomeadamente a idade, a educação e os recursos financeiros (Dush, Kotila, \& SchoppeSullivan, 2011; Hilton \& Devall, 1997; Stright \& Bales, 2003; van Egeren, 2003; Yárnoz-Yaben, 2010b). Da mesma forma, o ajustamento psicológico dos pais parece estar correlacionado com a coparentalidade. Por exemplo, Feinberg e Kahn (2008) encontraram que os sintomas depressivos e ansiosos em pais e mães estavam negativamente associados ao suporte coparental e positivamente relacionados com a sabotagem coparental. Tais resultados têm sido confirmados empiricamente por outros estudos (e.g., Bront-Tinkew, Moore, Matthews, \& Carrano, 2007; Dorsey et al., 2007; Yárnoz-Yaben, 2010b).

Globalmente, o ajustamento familiar é definido, por um lado, como o conjunto de padrões de comportamento que caracteriza a forma como cada um dos membros responde às suas funções (papéis familiares) e às necessidades da família e, por outro lado, como a competência de adaptação e flexibilidade estrutural e processual da família que lhe permite funcionar e gerir eficaz e eficientemente eventos ou transições causadoras de stress (Beavers \& Hampson, 2000; Epstein, Ryan, Bishop, Miller, \& Keitner, 2003). Conceptualmente, o funcionamento/ajustamento familiar está dinamicamente interdependente das características individuais e dos níveis de bem-estar psicológico dos seus membros, dos microprocessos familiares (tais como a conjugalidade, parentalidade, coparentalidade e relações entre irmãos) e de factores ecologicamente externos a si (e.g., ambiente e espaço comunitários, sistemas públicos de serviços sociais e de saúde; Epstein et al., 2003; Feinberg, 2003, Grzybowski \& Wagner, 2010). Mais concretamente, investigação prévia tem indicado que a aumentada sintomatologia depressiva nos pais pode estar negativamente relacionada com o funcionamento familiar (Conde, Figueiredo, \& Bifulco, 2011; Foster et al., 2008; 
Lamela, D., Figueiredo, B. \& Bastos, A. (2013). Perfis de Vinculação, Coparentalidade e Ajustamento Familiar em Pais RecémDivorciados: Diferenças no Ajustamento Psicológico.

Keitner, Ryan, Kohn, Bishop, \& Epstein, 1995), o aumento do conflito conjugal e o menor envolvimento parental (Cummings, Keller, \& Davies, 2005). Características sócio-demográficas parecem também contribuir para a predição do ajustamento familiar, tais como a idade (e.g., Pacheco, Costa, \& Figueiredo, 2009) e os recursos financeiros (R. Conger, K. Conger, \& Martin, 2010; Hilton \& Kopera-Frye, 2004; Papp, Cummings, \& Goeke-Morey, 2009).

Tomando como referência as famílias com pais divorciados, a conceptualização do ajustamento familiar incorpora as mudanças idiossincráticas produzidas pela dissolução conjugal no sistema familiar. Nesta linha, Portes, Smith, e Brown (2000), baseados na análise factorial do Divorce Adjustment Inventory, definem ajustamento familiar após o divórcio como baixo nível de conflito e disfunção familiares, presença de condições favoráveis na condução legal do divórcio e competências de coping dos filhos, resolução positiva do divórcio por parte dos pais, existência de apoio de sistemas externos de suporte e, por fim, a transição psicológica do divórcio. Na mesma linha, dados empíricos apontam para uma associação significativa entre o ajustamento de famílias binucleares e o ajustamento psicológico dos pais (Wang \& Amato, 2000). Por exemplo, Zimmerman, Brown e Portes (2004) encontraram que mães com a custódia dos filhos que apresentaram melhores níveis de funcionamento familiar exibiram, igualmente, menos sintomatologia psicopatológica. No entanto, o mesmo estudo não encontrou associações entre as variáveis sócio-demográficas e o ajustamento familiar (Zimmerman et al., 2004).

Considerando estes dados, a investigação teórica e empírica sugerem que o processo de reorganização da vinculação ao ex-cônjuge pode explicar a qualidade das relações coparentais e do funcionamento familiar neste período. As dimensões individual (vinculação), diádica (coparentalidade) e sistémica (ajustamento familiar) do processo de divórcio devem, por consequência, ser analisadas em conjunto (Dozier, Solie, \& Stack, 1993; Lamela, Figueiredo, et al., 2010). Alguns estudos encontraram uma associação entre a segurança de vinculação dos pais e a qualidade da coparentalidade. Em comparação com os pais com vinculação insegura evitante, os pais seguros demonstraram níveis mais elevados de coparentalidade após o divórcio; no entanto, não foram encontradas diferenças na coparentalidade entre os pais de vinculação segura e os pais de vinculação insegura ansiosa (Dozier et al., 1993; Figueiredo \& Lamela, 2011; Roberson, Nalbone, Hecker, \& Miller, 2010). Estes dados sugerem que a segurança na vinculação fornece aos pais divorciados um conjunto de estratégias de resolução problemas, competências de gestão das relações interpessoais e de regulação emocional perante eventos de distress que lhes permitem recorrer a coparentalidade com o intuito de promover o funcionamento ajustado dos filhos. Pelo contrário, os pais com vinculação insegura evitante, perante a inacessibilidade da figura de vinculação, recor- rem à desactivação das estratégias de vinculação, traduzindo-se na baixa qualidade da coparentalidade (mantida com a figura de vinculação; Figueiredo \& Lamela, 2011), o que vai de encontro com os pressupostos da teoria da vinculação (Cassidy \& Kobak, 1988).

Figueiredo e Lamela (2011) confirmando os resultados da investigação, demonstraram que não só a coparentalidade mas também o funcionamento familiar após o divórcio são explicados pela vinculação dos pais. Neste estudo, os autores encontraram que os pais seguros apresentavam maior percepção da qualidade da coparentalidade e maior ajustamento familiar. Os pais com uma vinculação ansiosa indicavam uma percepção da aliança estatisticamente idêntica aos pais de vinculação segura, no entanto com níveis mais baixos de ajustamento familiar. Por fim, os pais com uma vinculação insegura evitante demonstravam percepções mais reduzidas da coparentalidade do que os dois grupos, no entanto com um ajustamento familiar similar ao dos pais seguros e superior aos dos pais inseguros ansiosos. Para além de serem consistentes com a teoria da vinculação, estes resultados apontam para uma relação dinâmica entre factores individuais (vinculação), diádicos (coparentalidade) e sistémicos (funcionamento familiar) no processo de divórcio. Com a introdução da variável do ajustamento familiar, este estudo permitiu concluir que a manutenção da coparentalidade nos pais seguros e nos pais ansiosos pode ter funções diferentes. Enquanto, nos pais com vinculação segura, a coparentalidade funcionaria como um recurso para providenciar estabilidade e um funcionamento ajustado às suas famílias, nos pais com vinculação insegura ansiosa, a manutenção da coparentalidade desempenharia uma função de estratégia de hiperactivação perante a indisponibilidade da figura de vinculação após o divórcio. Considerando o baixo nível de ajustamento familiar, a coparentalidade pode ser usada pelos pais ansiosos, mais para enfatizar a sua vulnerabilidade $\mathrm{e}$ incapacidade e, dessa forma, procurar a atenção, protecção e cuidado do ex-cônjuge, do que propriamente para promover o ajustamento da família. Neste sentido, os dados de Figueiredo e Lamela (2011) sugerem que a coparentalidade para os pais ansiosos funciona mais como uma estratégia de procura de proximidade com a figura de vinculação do que como um recurso para a procura do bem-estar e funcionamento familiares.

Estas investigações têm o mérito de demonstrar que o funcionamento após o divórcio é complexo e deve ser analisado tendo em consideração os vários níveis da ecologia do comportamento humano (i.e., desde dimensões internas, como a vinculação, até dimensões sistémicas, como o funcionamento familiar). Consequentemente, o estudo do ajustamento psicológico dos adultos ao divórcio deve considerar esta análise multinível e não se basear apenas na investigação das dimensões individuais idiossincráticas a cada adulto divorciado. Além do divórcio ser um processo de reorganização da vinculação, a investigação sugere que se trata, igualmente, de um pro- 
cesso de reorganização diádica - ao nível da coparentalidade - e sistémica - ao nível do funcionamento familiar. Desta forma, utilizando parte substancial da amostra do estudo de Figueiredo e Lamela (2011), o presente artigo expande estes resultados com dois objectivos de investigação.

Como primeiro objectivo, pretendeu-se testar se perfis de regulação individual-diádico-sistémico após o divórcio podem ser identificados e confirmados empiricamente. Com o segundo objectivo pretendeu-se examinar diferenças ao nível do ajustamento psicológico e de variáveis sócio-demográficas dos pais récem-divorciados entre os perfis identificados no objectivo um.

\section{Método}

\section{Participantes}

A amostra foi constituída por 53 mães e 28 pais $(N=$ 81) que oficializaram o divórcio por mútuo consentimento numa Conservatória do Registo Civil de uma cidade do norte de Portugal ${ }^{1}$. Dois critérios de inclusão foram utilizados para a selecção dos participantes: ter pelo menos um filho com menos de 5 anos e não estar envolvido numa nova relação íntima. A média de idade dos participantes foi de 35,9 anos ( $D P=6,12$ anos). Na amostra, $27,4 \%$ dos participantes tinham 9 anos de escolaridade, $22,6 \%$ tinham 12 anos de escolaridade e $50 \%$ tinham graduação universitária. Noventa por cento dos participantes tinha um emprego com um rendimento médio anual de 32000 euros ( $D P=8360$ euros). Dos 81 participantes, 58 estiveram separados antes do divórcio legal $(M=4,92$ meses, $D P=7,56$ meses). Em média, os casamentos duraram 9,97 anos ( $D P=6,3$ anos). Quanto à regulação das responsabilidades parentais, $76,2 \%$ das guardas dos filhos era única, $21,4 \%$ guarda conjunta e $2,4 \%$ guarda alternada. Nas variáveis sócio-demográficas anteriores, não foram encontradas diferenças significativas entre as mães e os pais, com excepção na escolaridade, em que as mães tinham mais anos de escolaridade que os pais $t(2,79)=2,02, p<0,05$, e eram detentoras de mais guardas únicas, $t(2,79)=2,93, p<0,001$.

\section{Procedimentos}

A autorização e validação ética deste estudo foram concedidas pelos serviços centrais e locais da Conservatória do Registo Civil de Portugal. Aquando da oficialização legal do divórcio, as mães e os pais foram contactados nas instalações da Conservatória do Registo Civil por um investigador, a fim de fornecer informação sobre os objectivos do estudo. Apenas os pais que voluntariamente concordaram participar na investigação foram seleccionados. Após terem assinado o Termo de Consentimento Livre e Esclarecido, os participantes forneceram

\footnotetext{
${ }^{1}$ Em Portugal, os divórcios por mútuo consentimento podem ser celebrados sem a necessidade da presença de um juiz.
}

dados sócio-demográficos (e.g., nome, endereço e contacto telefónico). Foi entregue a cada participante a bateria de questionários (uma ficha sócio-demográfica e os questionários de auto-relato) e um envelope pré-pago. Foi igualmente solicitado que os participantes preenchessem os questionários em casa e os enviassem por correio (taxa de resposta de $81 \%$ ).

\section{Medidas}

Questionário Sócio-Demográfico. Este questionário tinha como finalidade recolher informação sobre as características sócio-demográficas dos participantes e dos filhos (e.g., género, idade, nível de escolaridade, rendimentos financeiros, anos de casamento, meses de separação prévia ao divórcio).

Vinculação dos Pais. Para avaliar a vinculação adulta foi administrado o Experiences in Close Relationships Scale (ECR; Brennan, Clark, \& Shaver, 1998). O ECR avalia duas dimensões da vinculação adulta: o evitamento à proximidade $\mathrm{e}$ a ansiedade ao abandono. $\mathrm{O}$ ECR é constituído por 36 itens numa escala tipo likert de 7 pontos, divididos em duas subescalas (evitamento e ansiedade). Este instrumento é um dos mais utilizados internacionalmente na avaliação da vinculação adulta, tendo sido já utilizado em amostras de adultos divorciados (e.g., Roberson et al., 2010). A versão portuguesa desta escala tem demonstrado níveis elevados de consistência interna, quer para o total da escala $(\alpha=0,86)$, quer para as subescalas: evitamento $(\alpha=0,88)$ e ansiedade $(\alpha=0,86$; Paiva \& Figueiredo, in press). Na interpretação dos resultados da escala, quanto maior o valor das subescalas, maior o evitamento e a ansiedade na vinculação e quanto menor o valor do total da escala, maior a segurança na vinculação adulta.

Coparentalidade. O Parenting Alliance Measure (PAM; Abidin \& Konold, 1999) foi utilizado para avaliar a percepção do progenitor sobre o grau de cooperação, comunicação e respeito mútuo da relação coparental que mantém com o outro pai. O PAM é um instrumento de 20 itens e foi utilizado em vários estudos, nomeadamente com pais divorciados (e.g., Bearss \& Eyberg, 1998). A versão portuguesa apresenta a mesma estrutura factorial que a versão original, apresentando níveis excelentes de consistência interna para o total da escala $(\alpha=0,92$; Lamela, Castro, Nunes-Costa, \& Figueiredo, 2011). No PAM, quanto maior a pontuação, maior a coparentalidade.

Ajustamento Familiar Pós-Divórcio. O Divorce Adjustment Inventory Revised (DAI-R; Portes et al., 2000) foi administrado para avaliar o ajustamento e funcionamento da família após a dissolução conjugal. Este instrumento de 42 itens e derivado empiricamente tem apresentado valor preditivo do funcionamento familiar e das crianças após o divórcio conjugal. As propriedades psicométricas da versão portuguesa são similares às da versão original, com os níveis de consistência interna a intervalar entre 0,65 a 0,84 (Lamela, Castro, \& Figueiredo, 2009). No 
Lamela, D., Figueiredo, B. \& Bastos, A. (2013). Perfis de Vinculação, Coparentalidade e Ajustamento Familiar em Pais RecémDivorciados: Diferenças no Ajustamento Psicológico.

presente estudo, apenas os valores do total do inventário foram utilizados. Nesta nova versão portuguesa do instrumento, quanto maior a pontuação no DAI-R, maior o ajustamento familiar após o divórcio.

Ajustamento Psicológico Parental. O Brief Symptom Inventory (BSI; Derogatis \& Melisaratos, 1983) foi utilizado para avaliar o ajustamento psicológico dos pais. O BSI é um instrumento de auto-relato com 53 itens, no qual o indivíduo classifica o grau em que cada problema listado o afectou durante a última semana. Através de uma escala de tipo likert, o ajustamento psicológico é avaliado em termos da ausência de sintomas psicopatológicos em 9 dimensões de sintomatologia (e.g., somatização, depressão e ansiedade) e três índices globais (Índice Geral de Sintomas - IGS, Total de Sintomas Positivos TSP e o Índice de Sintomas Positivos - ISP). No presente estudo, foram reportados os valores do IGS, por ser o melhor indicador único dos níveis actuais de distress/ ajustamento psicológico (Derogatis et al., 1983). A versão portuguesa do instrumento revelou que o inventário apresenta níveis adequados de consistência interna para as noves dimensões de sintomatologia e três índices (com valores de consistência interna desde 0,62 a 0,81 ). Segundo as normas de interpretação do BSI, quanto menor o valor do ISG, maior o ajustamento psicológico.

\section{Resultados}

\section{Perfis de Regulação Individual-Diádica-Sistémica}

A análise de clusters foi realizada procedendo a uma análise $K$-means (SPSS, versão 16 ), com distância Euclidiana quadrada como o índice de similaridade. As subescalas evitamento e ansiedade do ECR, o total das escalas PAM e DAI-R (totais estandardizados) foram utilizados na análise de clusters. O número de clusters foi determinado através da verificação do dendograma gerado a partir de uma análise de clusters hierárquica, usando o método de aglomeração hierárquica de Ward (1963). Esta análise conduziu à adopção de uma solução de 3 clusters, o que era consistente com resultados de investigações anteriores (e.g., Figueiredo \& Lamela, 2011; Roberson et al., 2010). A média dos scores dos participantes nas subescalas do ECR e nos totais do PAM e DAI-R são apresentados na Tabela 1.

Tabela 1

Valores Médios das Subescalas de Ansiedade e Evitamento do ECR, Total PAM e Total DAI-R para os Três Grupos

\begin{tabular}{lcccccccc}
\hline & $\begin{array}{c}\text { ECR } \\
\text { (Subescala } \\
\text { Ansiedade) }\end{array}$ & \multicolumn{2}{c}{$\begin{array}{c}\text { ECR } \\
\text { (Subescala } \\
\text { Evitamento) }\end{array}$} & & PAM & & DAI-R \\
\hline Grupo & $M$ & $D P$ & $M$ & $D P$ & $M$ & $D P$ & $M$ & $D P$ \\
\hline $\begin{array}{l}\text { Regulados-Seguros } \\
\quad(n=43)\end{array}$ & 3,57 & 0,60 & 2,37 & 0,93 & 71,16 & 14,78 & 148,07 & 10,35 \\
$\begin{array}{c}\text { Desregulados-Ansiosos } \\
(n=21)\end{array}$ & 4,79 & 0,72 & 2,37 & 0,64 & 69,52 & 16,03 & 127,67 & 10,82 \\
$\begin{array}{c}\text { Desregulados-Evitantes } \\
(n=17)\end{array}$ & 3,96 & 0,49 & 4,19 & 1,17 & 63,83 & 19,58 & 138,68 & 14,99 \\
$F \quad$ & 28,34 & 26,34 & & 33,33 & & 31,92 \\
\hline
\end{tabular}

Nota $. \mathrm{ECR}=$ Experience Close Relationship $; \mathrm{PAM}=$ Parenting Alliance Measure $; \mathrm{DAI}-\mathrm{R}=$ Divorce Adjustment Inventory Revised.

O cluster 1 ( $53 \%$ da amostra) tinha baixos valores médios nas subescalas de evitamento e ansiedade do ECR e os valores médios mais elevados nas escalas PAM e DAI-R. Dado que estes participantes apresentaram maior segurança na vinculação e maiores indicadores de ajustamento familiar e coparentalidade foram denominados por regulados-seguros. O cluster $2(26 \%$ da amostra $)$ tinha um valor médio elevado na subescala da ansiedade do ECR, um valor médio elevado no PAM e valores médios inferiores na subescala de evitamento do ECR e no total do DAI-R e foram denominados de desregulados-ansiosos. O cluster 3 (21\% da amostra) apresentou elevados valores médios na subescala de evitamento do ECR, baixos sinais de ansiedade na vinculação avaliada pela subescala ansiedade do ECR e baixos valores mé- dios no PAM e níveis médios no DAI-R. Consequen-temente, este cluster foi denominado por desreguladosevitantes.

A fim de identificar quais as variáveis com maior relevo na diferenciação dos 3 clusters retidos, foi analisada a estatística $F$ da ANOVA dos clusters (Burns \& Burns, 2008; Maroco, 2007). De acordo com Burns e Burns (2008), a magnitude dos valores $F$ realizados para cada uma das variáveis são um indicador de quão bem a variável analisada contribui para a discriminação entre cluters. Assim, a variável que parece permitir diferenciar mais os clusters é a coparentalida- de (PAM; $F=33,33)$, seguida pelo ajustamento familiar (medido através do DAI-R; $F$ $=31,92)$, pela ansiedade na vinculação (ECR, subescala ansiedade; $F=28,34$ ), sendo que a dimensão que menos 
contribui para a diferenciação dos clusters é o evitamento na vinculação (ECR, subescala evitamento; $F=26,34$; Tabela 1).

Comparações entre os Três Perfis nas Variáveis SócioDemográficas e no Ajustamento Psicológico Parental

Análises foram conduzidas para verificar diferenças entre os três grupos nas variáveis sócio-demográficas e no ajustamento psicológico parental. As análises de variância de um factor (ANOVAs) não relevaram diferenças estatisticamente significativas entre os três grupos em relação à idade média, ao rendimento médio anu$\mathrm{al}$, ao número de anos que durou o casamento e ao tempo de separação antes da legalização do divórcio (Tabela 2). Testes de $\chi^{2}$ não revelaram diferenças significativas na proporção de participantes em função do género, $\chi^{2}(2$, $N=81)=0,47, n$.s., e do tipo de guarda das crianças, $\chi^{2}$ $(2, N=81)=5,67$, n.s. . Tal como demonstrado na Tabela 2 , quando contrastado com os outros dois grupos, o perfil desregulados-evitantes apresentou níveis inferiores de anos de escolaridade.

Diferenças significativas foram encontradas entre os três grupos ao nível do ajustamento psicológico. O grupo regulado-seguro, quando comparado com os grupos desregulado-ansioso e desregulado-evitante, apresentou níveis mais elevados de ajustamento psicológico (i.e., valores menores no IGS). Não foram encontradas diferenças ao nível do ajustamento psicológico entre os grupos DA e DE, apesar dos valores de ajustamento psicológico serem significativamente inferiores ao grupo RS.

Tabela 2

Diferenças entre os Grupos (Perfis) de Regulação Individual-Diádica-Sistémica ao Nível das Variáveis SócioDemográficas e do Ajustamento Psicológico

\begin{tabular}{|c|c|c|c|c|c|c|c|c|c|c|}
\hline \multirow[b]{2}{*}{ Medidas } & \multicolumn{2}{|c|}{$\begin{array}{c}\mathrm{RS} \\
(n=43)\end{array}$} & \multicolumn{2}{|c|}{$\begin{array}{c}\text { DA } \\
(n=21)\end{array}$} & \multicolumn{2}{|c|}{$\begin{array}{c}\mathrm{DE} \\
(n=17)\end{array}$} & \multicolumn{4}{|c|}{ Testes de diferenças entre grupos } \\
\hline & $M$ & $D P$ & $M$ & $D P$ & $M$ & $D P$ & $g l$ & $F$ & $\eta^{2}$ & $\begin{array}{c}\text { Contrastes entre } \\
\text { grupos }^{\mathrm{a}}\end{array}$ \\
\hline Idade (anos) & 37,1 & 7,19 & 37,95 & 6,21 & 38,44 & 6,68 & 2,79 & 0,27 & 0,07 & n.s. \\
\hline $\begin{array}{l}\text { Rendimento médio } \\
\text { anual (euros) }\end{array}$ & 15940 & 8264 & 15540 & 8026 & 12210 & 8938 & 2,79 & 1,19 & 0,03 & n.s. \\
\hline Anos de escolaridade & 14 & 3,92 & 15,04 & 3,63 & 11,31 & 4,38 & 2,79 & $4,28 * *$ & 0,10 & $\mathrm{DE}<\mathrm{DA}, \mathrm{RS}$ \\
\hline Anos de casamento & 9,16 & 6,20 & 12,35 & 5,84 & 10,09 & 5,90 & 2,79 & 2,21 & 0,05 & n.s. \\
\hline $\begin{array}{l}\text { Meses de separação } \\
\text { pré-divórcio }\end{array}$ & $4,53^{\mathrm{b}}$ & 7,77 & $6,5^{\mathrm{c}}$ & 5,68 & $8,08^{\mathrm{d}}$ & 8,01 & 2,58 & 1,11 & 0,04 & n.s. \\
\hline IGS-BSI & 0,48 & 0,39 & 1,03 & 0,56 & 1,18 & 0,97 & 2,79 & $10,22 * * *$ & 0,24 & $\mathrm{DA}, \mathrm{DE}>\mathrm{RS}$ \\
\hline
\end{tabular}

Nota . RS = Regulados-seguros; DA = Desregulados-ansiosos; DE = Desregulados-evitantes; IGS-BSI = Índice global de sintomas do Brief Symptom Inventory (ajustamento psicológico).

${ }^{\mathrm{a}}$ Diferenças significativas entre grupos a $p<0,05$, usando o teste post-hoc de Scheffé; ${ }^{\mathrm{b}} n=30 ;{ }^{\mathrm{c}} n=16{ }^{\mathrm{d}} n=12$.

** $p<0,01 ; * * * p<0,001$.

\section{Discussão}

O presente estudo teve como objectivo identificar perfis de processo de divórcio, considerando a vinculação, a coparentalidade e o ajustamento familiar em pais recémdivorciados e sua relação com o seu ajustamento psicológico e variáveis sócio-demográficas. Apesar de tais variáveis surgirem frequentemente estudadas em investigações anteriores, não foram encontrados estudos na literatura que pesquisem a integração dinâmica destas três variáveis nas mesmas análises.

Investigações prévias sugeriam que pode ser adoptada uma análise multinível do processo do divórcio, congregando variáveis individuais, diádicas e sistémicas (Dozier et al., 1993; Figueiredo \& Lamela, 2011; Roberson et al., 2010). Os resultados significativos da análise de clusters sugerem que a interacção destas três variáveis multinível permitem identificar padrões específicos e circunscritos entre si do processo do divórcio.

Considerando o ajustamento psicológico dos pais recém-divorciados, os resultados mostraram que os pais regulados-seguros apresentavam melhor ajustamento psicológico (i.e., menor número de sintomas psicopatológicos) que os pais dos outros dois grupos. Estes resultados sugerem que o ajustamento psicológico no período imediatamente após a dissolução conjugal nos pais regulados-seguros é explicado por maiores níveis de segurança na vinculação e elevada coparentalidade e funcionamento familiar. Como estas três variáveis podem explicar os melhores valores de ajustamento psicológico deste grupo? Os resultados levam-nos a hipotetizar que os pais deste grupo, com vinculação segura adquiriram, ao longo da sua trajectória desenvolvimento, um sentido de competência interpessoal e desenvolveram padrões 
comportamentais, cognitivos e emocionais adaptativos e adequados, que lhes providenciam estratégias de coping capazes de responder às exigências desta transição familiar potencialmente causadora de distress e que funcionam como factor protector contra o desajustamento psicológico. Assim, a segurança da vinculação - resultado de baixos scores de ansiedade e evitamento na vinculação - parece permitir aos pais pertencentes a este grupo, por um lado, gerir adaptativamente a afectividade negativa associada à disrupção conjugal, valer-se do apoio emocional e instrumental da rede de suporte, o que resulta em valores mais elevados de ajustamento psicológico. Esta possível relação entre segurança na vinculação e o ajustamento psicológico pós-divórcio por nós levantada é corroborada por resultados de investigações anteriores (e.g., Davis et al., 2003; Tschann et al., 1989; YárnozYaben, 2010a).

$\mathrm{Na}$ mesma linha, os pais deste grupo percepcionaram a relação coparental como sendo caracterizada por melhor comunicação, mais baixos níveis de coercividade relacional, maior cooperação, suporte e partilha no cuidado dos filhos. Com base em estudos anteriores (DeGarmo, Patras, \& Eap, 2008; Dorsey et al., 2007; Meunier \& Roskam, 2009; Milkie, Bianchi, Mattingly, \& Robinson, 2002; Paulson, Dauber, \& Leiferman, 2009), este padrão coparental pode contribuir para menores níveis de afectividade negativa, menores níveis de stress, maior regulação emocional e maior sentimento de auto-eficácia. Consequentemente, tendo em conta que a educação dos filhos é vista como uma das tarefas a que os pais dedicam mais dos seus recursos no período pós-divórcio, levantamos a hipótese que o sentimento de apoio coparental por parte do ex-cônjuge e os menores níveis de afectividade negativa e conflito podem cooperar para melhores níveis de ajustamento psicológico, quer por via do suporte social percebido face a uma das tarefas mais exigentes desta transição, quer por via do impacto positivo que relações coparentais adequadas podem ter no ajustamento das crianças e das família. Neste sentido, a relação coparental parece funcionar como um recurso protector que facilita o ajustamento psicológico e instrumental dos pais deste grupo (Feinberg, 2003). Por outras palavras, a coparentalidade neste grupo pode funcionar não como um foco de instabilidade emocional, mas sim promotor do ajustamento psicológico.

Adicionalmente, o melhor ajustamento psicológico dos pais do perfil regulado-seguro são também explicados por melhores níveis de funcionamento familiar. Este resultado vai de encontro com dados de investigações anteriores que encontraram relações significativas entre dimensões do funcionamento familiar pós-divórcio e o ajustamento psicológico. Por exemplo, Rhode-Brown e Rudestam (2011) encontraram que adultos que perdoaram o excônjuge (resolução positiva do divórcio) apresentavam melhores níveis de ajustamento psicológico, enquanto DeGarmo et al. (2008) descobriram que o suporte social funciona como um factor protector para o ajustamento psicológico. Os nossos resultados em relação ao funcionamento familiar parecem sugerir que um adequado reajustamento das fronteiras relacionais entre os membros da família, a redefinição clara dos papéis familiares e o possível aumento da flexibilidade na gestão dos recursos internos e externos à família podem traduzir-se, por um lado, na diminuição de eventos potencialmente conflituosos dentro da família, na diminuição na ambiguidade quanto aos papéis esperados em cada um dos membros, promovendo trocas relacionais mais positivas e, por outro lado, no ajustamento psicossocial dos filhos, pode explicar o ajustamento psicológico dos pais em função do funcionamento familiar. Por exemplo, o estudo de Hilton e Kopera-Frye (2004) encontrou uma associação positiva significativa entre o funcionamento familiar e indicadores de ajustamento psicológico em pais e mães com a guarda dos filhos (satisfação com a vida e bemestar subjectivo), sendo que o funcionamento familiar apresentava-se como preditor dos níveis de depressão nos participantes do estudo. Neste sentido, sustentando-nos no modelo de resposta de ajustamento e adaptação familiar (Patterson, 1989, 2003), levantamos a suposição que a menor existência de focos de stress dentro da família e um adequado equilíbrio entre as exigências familiares associadas à transição do divórcio e as competências familiares (traduzidas nos recursos psicológicos e nos comportamentos de coping da família face a este evento stressor) nas famílias deste grupo contribuem para o ajustamento e adaptação da família, resultando no ajustamento psicológico dos pais.

Surpreendentemente, as dimensões que menos contribuíram para a diferenciação entre os clusters foram a ansiedade e o evitamento da vinculação. Teoricamente, estas variáveis reflectem os padrões de funcionamento cognitivo, emocional e comportamental dos indivíduos perante momentos de stress (Mikulincer \& Shaver, 2007), pelo que seria de esperar, numa perspectiva organicista, que a vinculação fosse a variável que melhor distinguisse os grupos. Todavia, a variável que melhor discriminou os grupos foi a coparentalidade precedida pelo ajustamento familiar. Assim, os nossos resultados parecem sugerir que uma análise individual-diádica-sistémica do processo de divórcio apresentou validade externa na compreensão desta transição e contribuir para hipóteses teóricas e dados da investigação contraditórios. Por exemplo, alguns autores (e.g., Dozier et al., 1993; Roberson et al., 2010) testaram a hipótese que a vinculação dos pais funcionar como um preditor da coparentalidade, enquanto outros investigadores verificaram a hipótese inversa, em que é a qualidade e frequência da coparentalidade após o divórcio predizia a vinculação dos adultos aos ex-parceiros (e.g., Madden-Derdich \& Arditti, 1999). No entanto, estas investigações anteriores corroboram parcialmente este argumento da interacção dinâmica entre factores individuais e diádicos no processo de divórcio.

Assim, como já acima referenciado, os nossos dados sugerem que estes perfis de regulação do processo de di- 
vórcio podem ser válidos na discriminação e na explicação da variação do ajustamento psicológico em pais recém-divorciados. Este dado assume relevância científica por duas razões. Primeiramente, aponta para a possibilidade do ajustamento psicológico dos pais não estar exclusivamente dependente de variáveis idiossincráticas dos adultos. Apesar de não ter sido testado e comparado o peso das variáveis idiossincráticas dos pais (e.g., estratégias de coping, suporte social) com o peso da interacção vinculação-coparentalidade-funcionamento familiar, os dados do nosso estudo levam a ponderar que o processo de ajustamento psicológico dos pais é um processo sistémico e familiarmente contextualizado. Finalmente, este resultado poderá apresentar importância nos contextos de aplicação, tais como nas intervenções clínicas individuais e sistémicas com adultos divorciados e com famílias com pais divorciados.

Apesar do seu contributo, o presente artigo deve ser analisado tendo em conta algumas precauções. Em primeiro, os resultados referem-se à avaliação da vinculação dos pais, coparentalidade e ajustamento familiar no período após o divórcio. Dada a natureza transversal deste estudo, dados teoricamente mais robustos seriam possíveis com um design metodológico longitudinal. Em segundo, os dados recolhidos circunscrevem-se a díades que se divorciaram por mútuo consentimento, subentendendo-se níveis baixos a moderados de conflito coparental. Considerando esta especificidade, estes resultados não devem ser lidos com generalizáveis a díades com divórcios litigiosos. Finalmente, devem ser sublinhados os elevados níveis de escolaridade e rendimentos médios da amostra estudada. É possível alguma variação dos resultados em função de níveis de educação e de rendimento inferiores.

Apesar destas limitações, o presente estudo oferece suporte empírico para a identificação de perfis de regulação do processo de divórcio baseados na vinculação, coparentalidade e ajustamento familiar, para além de fornecer base empírica para a possibilidade de o ajustamento psicológico ao divórcio ser compreendido por perfis de regulação individual-diádica-sistémica, contribuindo, desta forma, para a confirmação dos factores estruturais e processuais dentro da família funcionarem como variáveis endógenas na explicação do ajustamento psicológico dos pais a esta exigente transição familiar.

\section{Referências}

Abidin, R., \& Konold, T. (1999). Parenting Alliance Measure - Professional manual. Odessa, FL: Psychological Assessment Resources.

Bakermans-Kranenburg, M., \& Van IJzendoorn, M. (1997). Adult attachment and the break-up of romantic relationships. Journal of Divorce and Remarriage, 27, 121-141.

Bearss, K., \& Eyberg, S. (1998). A test of the parenting alliance theory. Early Education and Development, 9, 179-185.

Beavers, R., \& Hampson, R. (2000). The Beavers system model of family functioning. Journal of Family Therapy, 22, 128-143.
Brennan, K., Clark, C., \& Shaver, P. (1998). Self-report measurement of adult attachment: An integrative overview. In J. Simpson \& W. Rholes (Eds.), Attachment theory and close relationships (pp. 46-76). New York: Guilford Press.

Bront-Tinkew, J., Moore, K., Matthews, G., \& Carrano, J. (2007). Symptoms of major depression in a sample of fathers of infants: Sociodemographic correlates and links to father involvement. Journal of Family Issues, 28, 61-99.

Burns, R. B., \& Burns, R. A. (2008). Cluster analysis. Business research methods and statistics using SPSS. London: Sage.

Cassidy, J., \& Kobak, R. (1988). Avoidance and its relation to other defensive processes. In J. Belsky \& T. Nezworski (Eds.), Clinical implications of attachment (pp. 300-333). Hillsdale, NJ: Erlbaum.

Conde, A., Figueiredo, B., \& Bifulco, A. (2011). Attachment style and psychological adjustment in couples. Attachment \& Human Development, 13, 271-291.

Conger, R., Conger, K., \& Martin, M. (2010). Socioeconomic status, family processes, and individual development. Journal of Marriage and Family, 72, 685-704.

Cummings, E., Keller, P., \& Davies, P. (2005). Towards a family process model of maternal and paternal depression: Exploring multiple relations with child and family functioning. Journal of Child Psychology and Psychiatry, 46, 479-489.

Davis, D., Shaver, P., \& Vernon, M. (2003). Physical, emotional, and behavioral reactions to breaking up: The roles of gender, age, emotional involvement, and attachment style. Personality and Social Psychology Bulletin, 29, 871-884.

DeGarmo, D., Patras, J., \& Eap, S. (2008). Social support for divorced fathers' parenting: Testing a stress-buffering model. Family Relations, 57, 35-48.

Derogatis, L., \& Melisaratos, N. (1983). The Brief Symptom Inventory: An introductory report. Psychological Medicine, 13, 595-605.

Dorsey, S., Forehand, R., \& Brody, G. (2007). Coparenting conflict and parenting behavior in economically disadvantaged single parent African American families: The role of maternal psychological functioning. Journal of Family Violence, 22, 621-630.

Dozier, B., Solie, D., \& Stack, S. (1993). The effects of postdivorce attachment on coparenting relationships. Journal of Divorce \& Remarriage, 19, 109-124.

Dush, C., Kotila, L., \& Schoppe-Sullivan, S. (2011). Predictors of supportive coparenting after relationship dissolution among at-risk parents. Journal of Family Psychology, 25, 356-365.

Epstein, N., Ryan, C., Bishop, D., Millher, I., \& Keitner, G. (2003). The McMaster model: A view of healthy family functioning. In F. Walsh (Ed.), Normal family processes: Growing diversity and complexity ( $3^{\text {rd }}$ ed., pp. 581-606). New York: Guilford Press.

Feeney, B., \& Monin, J. (2008). An attachment-theoretical perspective on divorce. In J. Cassidy \& P. Shaver (Eds.), Handbook of attachment: Theory, research, and clinical applications ( $2^{\text {nd }}$ ed., pp. 934-957). New York: Guilford Press.

Feinberg, M. (2003). The internal structure and ecological context of coparenting: A framework for research and intervention. Parenting, 3, 85-131.

Feinberg, M., \& Kan, M. (2008). Establishing family foundations: Intervention effects on coparenting, parent/infant well-being, and parent-child relations. Journal of Family Psychology, 22, 253-263.

Figueiredo, B., \& Lamela, D. (2011). Attachment, parenting alliance and post-divorce family adjustment. Manuscript submited for publication. 
Lamela, D., Figueiredo, B. \& Bastos, A. (2013). Perfis de Vinculação, Coparentalidade e Ajustamento Familiar em Pais RecémDivorciados: Diferenças no Ajustamento Psicológico.

Foster, C., Webster, M., Weissman, M., Pilowsky, D., Wickramaratne, P., Rush, J., et al. (2008). Course and severity of maternal depression: Associations with family functioning and child adjustment. Journal of Youth and Adolescence, 37, 906-916.

Gähler, M. (2006). "To divorce is to die a bit...": A longitudinal study of marital disruption among Swedish women and men. The Family Journal, 14, 372-382.

Grzybowski, L., \& Wagner, A. (2010). O envolvimento parental após a separação/divórcio. Psicologia: Reflexão e Crítica, 23, 289-298.

Hilton, J., \& Devall, E. (1997). The Family Economic Strain Scale: Development and evaluation of the instrument with single - And two-parent families. Journal of Family and Economic Issues, 18, 247-271.

Hilton, J., \& Kopera-Frye, K. (2004). Patterns of psychological adjustment among divorced custodial parents. Journal of Divorce \& Remarriage, 41, 1-30.

Keitner, G., Ryan, C., Kohn, R., Bishop, D., \& Epstein, N. (1995). Role of family in recovery and major depression. American Journal of Psychiatry, 152, 1002-1008.

Kitson, G. (1982). Attachment to the spouse in divorce: A scale and its application. Journal of Marriage and Family, 44, 379-393.

Lamela, D., Castro, M., \& Figueiredo, B. (2009). Psychometric properties of Portuguese version of the Divorce Adjustment Inventory Revised. Unpublished manuscript, University of Minho, Braga, Portugal.

Lamela, D., Castro, M., Nunes-Costa, R., \& Figueiredo, B. (2011). Medida da Aliança Parental: Validação portuguesa e construção de uma versão reduzida. Manuscrito submetido para publicação.

Lamela, D., Figueiredo, B., \& Bastos, A. (2010). Adaptação ao divórcio e relações coparentais: Contributos da teoria da vinculação. Psicologia: Reflexão e Crítica, 23, 562-574.

Lamela, D., Nunes-Costa, R., \& Figueiredo, B. (2010). Modelos teóricos das relações coparentais: Revisão crítica. Psicologia em Estudo, 15, 205-216.

Lansford, J. (2009). Parental divorce and children's adjustment. Perspectives on Psychological Science, 4, 140-152.

Madden-Derdich, D., \& Arditti, J. (1999). The ties that bind: Attachment between former spouses. Family Relations, 48, 243-249.

Madden-Derdich, D., Leonard, S., \& Christopher, F. (1999). Boundary ambiguity and coparental conflict after divorce: An empirical test of a family systems model of the divorce process. Journal of Marriage and the Family, 61, 588-598.

Maroco, J. (2007). Análise de clusters. Análise estatística com a utilização do SPSS (3. ed.). Lisboa, Portugal: Edições Sílabo.

McHale, J., \& Irace, K. (2011). Coparenting in diverse family systems. In J. McHale \& K. Lindahl (Eds.), Coparenting: A conceptual and clinical examination of family systems (pp. 15-37). Washington, DC: American Psychological Association.

McHale, J., \& Lindahl, K. (2011). What is coparenting? In J. McHale \& K. Lindahl (Eds.), Coparenting: A conceptual and clinical examination of family systems (pp. 3-12). Washington, DC: American Psychological Association.

Meunier, J., \& Roskam, I. (2009). Self-efficacy beliefs amongst parents of young children: Validation of a self-report measure. Journal of Child and Family Studies, 18, 495-511.
Mikulincer, M., \& Shaver, P. (2007). Attachment patterns in adulthood: Structure, dynamics, and change. New York: Guilford Press.

Milkie, M., Bianchi, S., Mattingly, M., \& Robinson, J. (2002). Gendered division of childrearing: Ideals, realities, and the relationship to parental well-being. Sex Roles, 47, 21-38.

Minuchin, P. (1974). Families and family therapy. Cambridge, MA: Harvard University Press.

Nunes-Costa, R., Lamela, D., \& Figueiredo, B. (2009). Psychosocial adjustment and physical health in children of divorce. Jornal de Pediatria, 85, 385-396.

Pacheco, A., Costa, R., \& Figueiredo, B. (2009). Qualidade do relacionamento com pessoas significativas: Comparação entre grávidas adolescentes e adultas. Psicologia: Teoria e Prática, 11, 129-144.

Paiva, C., \& Figueiredo, B. (in press). Versão portuguesa do questionário Experiences in Close Relationships (ECR). In M. Simões, L. Almeida, M. Gonçalves, \& C. Machado (Eds.), Avaliação Psicológica: Vol. IV. Instrumentos validados para a população portuguesa. Coimbra, Portugal: Quarteto Editora.

Papp, L., Cummings, E., \& Goeke-Morey, M. (2009). For richer, for poorer: Money as a topic of marital conflict in the home. Family Relations, 58, 91-103.

Patterson, J. (1989). A family stress model: The family adjustment and adaptation response. In C. Ramsey (Ed.), The science of family medicine (pp. 95-118). New York: Guilford Press.

Patterson, J. (2003). Understanding family resilience. Journal of Clinical Psychology, 58, 233-246.

Paulson, J., Dauber, S., \& Leiferman, J. (2009). Parental depression, relationship quality, and nonresident father involvement with their infants. Journal of Family Issues, 32, 528-549.

Portes, P., Smith, T., \& Brown, J. (2000). The divorce adjustment inventory-revised: Validation of a parental report concerning children's post-custody adjustment. Journal of Divorce \& Remarriage, 33, 93-109.

Raposo, H., Figueiredo, B., Lamela, D., Nunes-Costa, R., Castro, M., \& Prego, J. (2011). Ajustamento da criança à separação ou divórcio dos pais. Psiquiatria Clínica, 38, 29-33.

Rhode-Brown, J., \& Rudestam, K. E. (2011). The role of forgiveness in divorce adjustment and the impact of affect. Journal of Divorce and Remarriage, 52(2), 109-124.

Roberson, P., Nalbone, D., Hecker, L., \& Miller, J. (2010). Adult attachment style as a predictor of coparent relationships postdivorce. Journal of Divorce \& Remarriage, 51, 185-207.

Schoppe, S., Mangelsdorf, S., \& Frosch, C. (2001). Coparenting, family process and family structure: Implications for preschoolers' externalizing behavior problems. Journal of Family Psychology, 15, 526-545.

Schoppe-Sullivan, S., Mangelsdorf, S., Frosch, C., \& McHale, J. (2004). Associations between coparenting and marital behavior from infancy to the preschool years. Journal of Family Psychology, 18, 194-207.

Shaver, P., \& Mikulincer, M. (2007). Adult attachment strategies and the regulation of emotion. In J. J. Gross (Ed.), Handbook of emotion regulation (pp. 446-465). New York: Guilford Press.

Stright, A., \& Bales, S. (2003). Coparenting quality: Contributions of child and parent characteristics. Family Relations, $52,232-240$. 
Teubert, D., \& Pinquart, M. (2010). The association between coparenting and child adjustment: A meta-analysis. Parenting, 10, 286-307.

Tschann, J., Johnston. J., \& Wallerstein, J. (1989). Resources, stressors, and attachment as predictors of adult adjustment after divorce: A longitudinal study. Journal of Marriage and the Family, 51, 1033-1046.

van Egeren, L. (2003). Pre-birth predictors of coparenting experience trajectories in early infancy. Infant Mental Health Journal, 24, 278-295.

van Egeren, L. (2004). The development of coparenting over the transition to parenthood. Infant Mental Health Journal, 25, 453-477.

Wang, H., \& Amato, P. (2000). Predictors of divorce adjustment: Stressors, resources, and definitions. Journal of Marriage and Family, 62, 655-668.

Ward, J. H. (1963). Hierarchical grouping to optimize an objective function. Journal of the American Statistical Association, 58, 236-244.

Yárnoz-Yaben, S. (2010a). Attachment style and adjustment to divorce. The Spanish Journal of Psychology, 13, 210-219.

Yárnoz-Yaben, S. (2010b). Hacia la coparentalidad post-divorcio: percepción del apoyo de la ex pareja en progenitores divorciados españoles. International Journal of Clinical and Health Psychology, 10, 295-307.

Zimmerman, D., Brown, J., \& Portes, P. (2004). Assessing custodial mother adjustment to divorce: The role of divorce education and family functioning. Journal of Divorce \& Remarriage, 41, 1-24. 